\title{
High prevalence of PIK3CA/AKT pathway mutations in papillary neoplasms of the breast
}

\author{
Megan L Troxell ${ }^{1}$, Judith Levine ${ }^{1,2}$, Carol Beadling ${ }^{1}$, Andrea Warrick ${ }^{1}$, Jennifer Dunlap ${ }^{2}$, \\ Ajia Presnell ${ }^{1}$, Janice Patterson ${ }^{1}$, Arielle Shukla ${ }^{1,2}$, Neal R Olson ${ }^{3}$, Michael C Heinrich ${ }^{1,4,5}$ \\ and Christopher L Corless ${ }^{1,2}$
}

${ }^{1}$ Knight Cancer Institute, Oregon Health \& Science University, Portland, OR, USA; ${ }^{2}$ Department of Pathology, Oregon Health \& Science University, Portland, OR, USA; ${ }^{3}$ Department of Pathology, Kaiser Permanente Northwest, Portland, OR, USA; ${ }^{4}$ Portland Veterans Affairs Medical Center, Portland, OR, USA and ${ }^{5}$ Division of Hematology and Medical Oncology, Department of Medicine, Oregon Health \& Science University, Portland, OR, USA

\begin{abstract}
Papillary lesions of the breast have an uncertain relationship to the histogenesis of breast carcinoma, and are thus diagnostically and managerially challenging. Molecular genetic studies have provided evidence that ductal carcinoma in situ and even atypical ductal hyperplasia are precursors of invasive carcinoma. However, papillary lesions have been seldom studied. We screened papillary breast neoplasms for activating point mutations in PIK3CA, AKT1, and RAS protein-family members, which are common in invasive ductal carcinomas. DNA extracts were prepared from sections of 89 papillary lesions, including 61 benign papillomas (28 without significant hyperplasia; 33 with moderate to florid hyperplasia), 11 papillomas with atypical ductal hyperplasia, 7 papillomas with carcinoma in situ, and 10 papillary carcinomas. Extracts were screened for PIK3CA and AKT1 mutations using mass spectrometry; cases that were negative were further screened for mutations in AKT2, BRAF, CDK, EGFR, ERBB2, KRAS, NRAS, and HRAS. Mutations were confirmed by sequencing or HPLC assay. A total of 55 of 89 papillary neoplasms harbored mutations (62\%), predominantly in AKT1 (E17K, 27 cases) and PIK3CA (exon 20 >exon 9, 27 cases). Papillomas had more mutations in AKT1 (54\%) than in PIK3CA (21\%), whereas papillomas with hyperplasia had more PIK3CA $(42 \%)$ than AKT1 (15\%) mutations, as did papillomas with atypical ductal hyperplasia (PIK3CA 45\%, AKT1 27\%, and NRAS 9\%). Among seven papillomas with carcinoma in situ, three had AKT1 mutations. The 10 papillary carcinomas showed an overall lower frequency of mutations, including 1 with an $A K T 1$ mutation (in a tumor arising from a papilloma), 1 with an NRAS gene mutation (Q61H), and 2 with PIK3CA mutations (1 overlapping with the NRAS Q61H). These findings indicate that approximately two-thirds of papillomas are driven by mutations in the PI3CA/AKT pathway. Some papillary carcinomas may arise from these lesions, but others may have different molecular origins.
\end{abstract}

Modern Pathology (2010) 23, 27-37; doi:10.1038/modpathol.2009.142; published online 6 November 2009

Keywords: phosphoinositol-3-kinase (PIK3CA); AKT1; breast; papilloma; papillary carcinoma

Papillary lesions of the breast are quite common, but remain enigmatic in terms of their pathogenesis and managerial implications. Papillary breast lesions are characterized by fibrovascular stalks lined by

Correspondence: Dr ML Troxell, MD, PhD, Department of Pathology, Oregon Health \& Science University, 3181 SW Sam Jackson Park Rd, L471, Portland, OR 97239, USA.

E-mail: troxellm@ohsu.edu

Received 2 June 2009; revised 8 July 2009; accepted 21 July 2009; published online 6 November 2009 epithelial cells, residing in cystically dilated ductal spaces. Papillomas are lined by two cell types: myoepithelial cells situated along a basement membrane and overlying epithelial cells, which may harbor any architectural or cytologic arrangement seen elsewhere in the breast, including mild to florid hyperplasia, columnar cell change, atypical ductal hyperplasia, ductal carcinoma in situ, or lobular neoplasia. In contrast, papillary carcinoma is conventionally defined by the lack of myoepithelial cells along fibrovascular stalks. Historical 
studies show that papillomas lacking cytologic atypia are associated with an approximately twofold risk of subsequent breast cancer, similar to that seen with usual ductal hyperplasia/proliferative fibrocystic disease. ${ }^{1}$ However, the management of papillary lesions on needle core biopsy is difficult given the potential for epithelial variability across such lesions (sampling artifact), and there remains poor understanding of the pathogenetic implications of papillary lesions.

Relatively few studies have characterized molecular or cell-biologic aberrations in papillary lesions of the breast. Noguchi and colleagues ${ }^{2,3}$ showed that papillomas are clonal by studying patterns of $\mathrm{X}$-inactivation, thus allowing their classification as papillary neoplasms. Several subsequent studies looking at large-scale chromosomal alterations (using methods of fluorescence in situ hybridization, loss of heterozygosity, and comparative genomic hybridization) have generated conflicting data regarding the presence or frequency of alterations in benign papillary lesions. ${ }^{4-9}$ Thus, it is unclear whether papillary neoplasms harbor genetic changes common to the pathways of breast cancer progression, and it remains undetermined whether papillary lesions represent precursors of breast carcinoma.

Recent studies have shown that point mutations in known tumor-suppressor genes and protein kinases are present in some breast cancers; however, they have been little studied in proliferative or precursor lesions. The gene encoding the phosphoinositol-3-kinase catalytic subunit (PIK3CA) is one of the most frequently mutated genes in breast carcinoma, with activating mutations identified in approximately one-quarter of studied cases (range $8-40 \%$ ), and these mutations are present at the stage of ductal carcinoma in in situ. ${ }^{10-20}$ The vast majority of mutations are located in 'hotspots' in exon 9 (helical domain) and exon 20 (kinase domain), with small numbers of mutations identified in other exons. ${ }^{10-20}$ Phosphoinositol-3-kinase is activated in response to receptor-tyrosine kinase signaling at the cell membrane, and acts through a cascade to phosphorylate AKT, itself a kinase. Activating mutations in the pleckstrin-homology domain of $A K T 1$ have been identified in approximately $4 \%$ of invasive breast cancers (range 1.5-8\%). ${ }^{19,21-23}$ Activation of the PIK3CA/AKT pathway signals through mTOR to promote protein translation and cell cycling, and other effectors participate in regulation of transcription, apoptosis, cellular metabolism, etc. ${ }^{11,24-26}$ PIK3CA/AKT signaling is engaged in cross talk with several intracellular cascades, including RAS and estrogen receptor pathways. ${ }^{11,24-26}$ Given the frequency of PIK3CA and AKT1 mutations in invasive and in situ breast carcinoma, we investigated the mutational status of papillary neoplasms as a means to study their pathogenetics, and thus their role as putative precursor lesion in breast carcinogenesis.

\section{Materials and methods}

\section{Papillary Lesional Tissue}

This study was approved by the institutional review boards at Oregon Health \& Science University, and Kaiser Permanente Northwest, Portland, OR. The computerized files of Oregon Health \& Science University (2000-2008) and Kaiser Permanente Northwest (2005-2008) were searched for breast specimens containing papillary lesions; core needle biopsies were excluded. All cases were reviewed by a pathologist with interest in breast pathology (MLT), and appropriate blocks from cases with adequate lesional tissue were selected. The greatest cross-sectional diameter was measured on representative slides (to the nearest $\mathrm{mm}$, perpendicular to the involved duct).

Papillary lesions were evaluated histologically and categorized as follows. Papillomas were defined as arborizing fibrovascular stalks lined by at least two layers (myoepithelium and epithelium) of nonatypical epithelial cells, within dilated ductal/ cystic space. Papillomas with atypical ductal hyperplasia were defined as papillomas in which the overlying epithelial proliferation showed cytologic atypia, but did not meet cytologic and architectural criteria for ductal carcinoma in situ $;^{27}$ papillomas with ductal carcinoma in situ were diagnosed without regard to proposed size criteria. ${ }^{28}$ Papillary carcinoma was defined as a papillary proliferation lacking myoepithelial cells along papillary fibrovascular stalks. For the purposes of the study, lesions showing focal loss of myoepithelial cells were diagnosed as papillary carcinoma arising in a papilloma, and we used p63, calponin, or smooth muscle myosin heavy-chain immunohistochemical staining to highlight myoepithelial cells in select cases. ${ }^{1,28,29}$ Solid papillary carcinoma was defined as described by Maluf and Koerner., ${ }^{1,28-30}$

In addition, the extent of epithelial hyperplasia was recorded as mild (epithelium 2-3 layers thick), moderate (epithelium $>3$ layers thick), or florid (spaces between fibrovascular cores completely filled by epithelial proliferation) using standard criteria, ${ }^{31}$ and the presence of columnar cell change or apocrine metaplasia was also recorded. One nipple duct adenoma with moderate hyperplasia was also included (benign papillary category). The presence of in situ or invasive carcinoma elsewhere in the breast specimen, not involving the papillary lesion, was noted.

\section{Preparation of DNA Extracts}

Formalin-fixed paraffin-embedded tissue sections were cut at $7 \mu \mathrm{m}$ onto glass slides. Using an H\&Estained section as template, areas of papillary lesion were dissected using a clean scalpel blade, then deparaffinized with successive xylene and ethanol 
washes. DNA was extracted using the Qiagen DNA Mini Kit (Qiagen, Valencia, CA, USA).

\section{Mutation Screening}

The Sequenom MassARRAY system was used to screen for mutations. In brief, this system involves PCR amplification of sequences of interest, followed by primer extension and mass spectrometry (MALDI-TOF MS) as previously detailed. ${ }^{32}$ Assays were derived from the Sequenom OncoCarta panel (Sequenom, San Diego, CA, USA) and were validated in the laboratory. The majority of cases were first run on a mini panel encompassing mutation assays for PIK3CA exon 4 (N545K) and exon 20 (H1047R/L), and AKT1 exon 2 (E17K); cases without identified mutations were then screened with the full OncoCarta panel. PCR-amplified DNA was cleaned using EXO-SAP (Sequenom), and primer was extended by TYPLEX chemistry, desalted using Clean Resin (Sequenom), and spotted onto SpectroCHIP II matrix chips using the MassARRAY Nanodispenser RS1000 (Sequenom). Chips were run on a Sequenom MassARRAY MALDI-TOF system. Sequenom Typer software and visual inspection were used to interpret mass spectra. Four cases were not screened on Sequenom, but were primarily analyzed by HPLC WAVE and direct sequencing (two cases of papilloma with ductal carcinoma in situ, one papillary carcinoma, one nipple duct adenoma, see below). Four cases that failed to spot on the Sequenom chip were assayed similarly (one case each of papilloma, papilloma with hyperplasia, papilloma with atypical ductal hyperplasia, and papilloma with ductal carcinoma in situ). There was no difference in size of papillary lesion in cases assayed as wild type vs mutant.

\section{Confirmation of Mutations}

PIK3CA exons 9 and 20, and AKT1 exon 2 were amplified by PCR using primers as previously described, using TAQ polymerase (Expand High Fidelity PCR System, Roche, Indianapolis, IN, USA), with parallel positive and negative controls. ${ }^{20}$ PCR products were run on a Transgenomic WAVE HPLC system (Transgenomic, Omaha, NE, USA; melting temperature determined empirically for each primer pair). ${ }^{20}$

Alternatively, mutations were confirmed by direct sequencing on an ABI3130 sequencer using the BigDye Terminator method.

\section{Immunohistochemical Staining}

Unstained sections ( $5 \mu \mathrm{m}$ ) were prepared on charged glass slides, and stained immunohistochemically for estrogen receptor using standard protocols on a Ventana BenchMark XT automated platform
(Ventana, Tucson, AZ, USA) with rabbit monoclonal antibody SP1 (Ventana). Results were scored semiquantitatively blinded to gene mutation status (by MLT), including both intensity ( 0 , negative; $1+$, weak; $2+$, moderate; and $3+$, strong) and estimated percentage of nuclei labeled. A product score was calculated by multiplying percent positive nuclei by intensity.

\section{Statistical Analysis}

The correlation of PIK3CA and/or AKT1 mutational status to histologic type was analyzed by Fisher's exact test (two-tailed) using the Statview program (SAS Institute, Inc., Cary, NC, USA). A $P$-value of 0.05 was considered significant.

\section{Results}

\section{Papillary Lesions}

Papillary breast lesions with adequate tissue for analysis were identified from the files of Oregon Health \& Science University and Kaiser Permanente Northwest. The study group consisted of 89 papillary lesions, including 61 papillomas without atypia or carcinoma; of these 33 had moderate or florid epithelial hyperplasia. Eighteen papillary lesions had atypia within the epithelial component, including 11 papillomas with atypical ductal hyperplasia, 6 with ductal carcinoma in situ, and 1 involved by lobular carcinoma in situ. There were 10 papillary carcinomas, including 3 carcinomas arising within papillomas. Three cases had features of the solid variant of papillary carcinoma, including one arising within a papilloma, and two with invasion. Four of the papillary carcinomas were accompanied by an invasive component, including one with tubulopapillary features and one microinvasive $(<1 \mathrm{~mm}$ invasion). The cross-sectional diameter of papillary lesions ranged from 0.1 to $1.5 \mathrm{~cm}$, average $0.4 \mathrm{~cm}$.

\section{Mutational Analysis}

Papillary neoplasms were screened for hotspot point mutations in AKT1, PIK3CA, KRAS, HRAS, and NRAS using mass spectroscopy. Mutations were confirmed with PCR followed by HPLC assays, or direct sequencing. Eight cases were screened primarily by HPLC, with sequence confirmation. In one case, confirmatory analysis was unsuccessful; in all others there was complete concordance with the initial mass spectroscopy result.

Of 89 tested papillary lesions, we identified $A K T 1$ E17K mutations in 27 cases (30\%, Tables 1 and 2, Figures 1 and 2). A further $21(24 \%)$ cases had PIK3CA exon 20 kinase domain mutations, including $18 \mathrm{H} 1047 \mathrm{R}$ and $3 \mathrm{H} 1047 \mathrm{~L}$ mutations (Tables 1 and 2, Figures 1 and 2). Interestingly, we identified only four cases (5\%) with PIK3CA exon 9 helical 
Table 1 Mutational analysis of papillary neoplasms

\begin{tabular}{|c|c|c|}
\hline Case & Diagnosis & Mutation \\
\hline \multicolumn{3}{|c|}{ AKT1 exon 2} \\
\hline 5 & Papilloma & AKT1 E17K \\
\hline 14 & Papilloma & AKT1 E17K \\
\hline 19 & Papilloma & AKT1 E17K \\
\hline 25 & Papilloma & AKT1 E17K \\
\hline 30 & Papilloma & AKT1 E17K \\
\hline 32 & Papilloma & AKT1 E17K \\
\hline 33 & Papilloma & AKT1 E17K \\
\hline 39 & Papilloma & AKT1 E17K \\
\hline 46 & Papilloma & AKT1 E17K \\
\hline 48 & Papilloma & AKT1 E17K \\
\hline 49 & Papilloma & AKT1 E17K \\
\hline 51 & Papilloma & AKT1 E17K \\
\hline 52 & Papilloma & AKT1 E17K \\
\hline 53 & Papilloma & AKT1 E17K \\
\hline 62 & Papilloma & AKT1 E17K \\
\hline 6 & Papilloma, hyperplasia & AKT1 E17K \\
\hline 40 & Papilloma, hyperplasia & AKT1 E17K \\
\hline 47 & Papilloma, hyperplasia & AKT1 E17K \\
\hline 60 & Papilloma, hyperplasia & AKT1 E17K \\
\hline 67 & Papilloma, hyperplasia & AKT1 E17K \\
\hline 15 & Papilloma, atypical ductal hyperplasia & AKT1 E17K \\
\hline 23 & Papilloma, atypical ductal hyperplasia & AKT1 E17K \\
\hline 34 & Papilloma, atypical ductal hyperplasia & AKT1 E17K \\
\hline 74 & Papilloma, ductal carcinoma in situ & AKT1 E17K \\
\hline 80 & Papilloma, ductal carcinoma in situ & AKT1 E17K \\
\hline 78 & Papilloma, ductal carcinoma in situ & AKT1 E17K \\
\hline 86 & Papillary carcinoma arising in papilloma & AKT1 E17K \\
\hline \multicolumn{3}{|c|}{ PIK3CA exon 4} \\
\hline 38 & Papilloma, hyperplasia & PIK3CA N345K \\
\hline \multicolumn{3}{|c|}{ PIKЗСА exon 9} \\
\hline 7 & Papilloma & PIK3CA E542K \\
\hline 56 & Papilloma & PIK3CA E545K \\
\hline 45 & Papilloma, hyperplasia & PIK3CA E545K \\
\hline 89 & Papillary carcinoma & PIK3CA E542K \\
\hline \multicolumn{3}{|c|}{ PIKЗCA exon 13} \\
\hline 87 & Papillary carcinoma, invasive (tubulopapillary) & PIK3CA H701P, E707K, L719V (and NRAS) \\
\hline \multicolumn{3}{|c|}{ PIK3CA exon 20} \\
\hline 1 & Papilloma & PIK3CA H1047L \\
\hline 16 & Papilloma & PIK3CA H1047R \\
\hline 35 & Papilloma & PIK3CA H1047R \\
\hline 58 & Papilloma & PIK3CA H1047R \\
\hline 9 & Papilloma, hyperplasia & PIK3CA H1047R \\
\hline 18 & Papilloma, hyperplasia & PIK3CA H1047R \\
\hline 26 & Papilloma, hyperplasia & PIK3CA H1047R \\
\hline 28 & Papilloma, hyperplasia & PIK3CA H1047R \\
\hline 29 & Papilloma, hyperplasia & PIK3CA H1047R \\
\hline 36 & Papilloma, hyperplasia & PIK3CA H1047R \\
\hline 37 & Papilloma, hyperplasia & PIK3CA H1047R \\
\hline 50 & Papilloma, hyperplasia & PIK3CA H1047R \\
\hline 54 & Papilloma, hyperplasia & PIK3CA H1047R \\
\hline 61 & Papilloma, hyperplasia & PIK3CA H1047R \\
\hline 65 & Papilloma, hyperplasia & PIK3CA H1047R \\
\hline 66 & Papilloma, hyperplasia & PIK3CA H1047R \\
\hline 13 & Papilloma, atypical ductal hyperplasia, & PIK3CA H1047R \\
\hline 24 & Papilloma, atypical ductal hyperplasia & PIK3CA H1047L \\
\hline 69 & Papilloma, atypical ductal hyperplasia & PIK3CA H1047R \\
\hline 73 & Papilloma, atypical ductal hyperplasia & PIK3CA H1047R \\
\hline 77 & Papilloma, atypical ductal hyperplasia & PIK3CA H1047L \\
\hline \multicolumn{3}{|c|}{ RAS family mutations } \\
\hline 76 & Papilloma, atypical ductal hyperplasia & HRAS G12D \\
\hline 87 & Papillary carcinoma, invasive (tubulopapillary) & NRAS Q61H (and PIK3CA exon 13) \\
\hline
\end{tabular}


Table 2 Frequency of mutations by type of papillary lesion

\begin{tabular}{|c|c|c|c|c|c|c|c|}
\hline Lesion & $\begin{array}{c}A K T 1 \\
(\%)\end{array}$ & $\begin{array}{c}\text { PIK3CA All } \\
\text { exons (\%) }\end{array}$ & $\begin{array}{c}\text { PIK3CA } \\
\text { exon } 9(\%)\end{array}$ & $\begin{array}{c}\text { PIK3CA } \\
\text { exon } 20(\%)\end{array}$ & $\begin{array}{c}\text { PIK3CA } \\
4,13(\%)\end{array}$ & $\begin{array}{l}R A S \\
(\%)\end{array}$ & $\begin{array}{l}\text { Wild type } \\
(\%)\end{array}$ \\
\hline Papilloma without atypia $(\mathrm{N}=61)$ & $20(33 \%)$ & $20(33 \%)$ & $3(5 \%)$ & $16(26 \%)$ & $1(2 \%)$ & $\mathbf{0}(\mathbf{0} \%)$ & $21(34 \%)$ \\
\hline No epithelial hyperplasia $(N=28)$ & $15(54 \%)$ & $6(21 \%)$ & $2(7 \%)$ & $4(14 \%)$ & $0(0 \%)$ & & $7(25 \%)$ \\
\hline Moderate-florid hyperplasia $(N=33)$ & $5(15 \%)$ & $14(42 \%)$ & $1(3 \%)$ & $12(36 \%)$ & $1(3 \%)$ & & $14(42 \%)$ \\
\hline Papilloma with atypia, CIS $(\mathrm{N}=18)$ & $6(33 \%)$ & $5(28 \%)$ & $0(0 \%)$ & $5(28 \%)$ & $0(0 \%)$ & $1(6 \%)$ & $6(33 \%)$ \\
\hline $\mathrm{ADH}(N=11)$ & $3(27 \%)$ & $5(45 \%)$ & & $5(45 \%)$ & & $1(9 \%)$ & $2(19 \%)$ \\
\hline DCIS $(N=6)$ & $3(50 \%)$ & 0 & & 0 & & 0 & $3(50 \%)$ \\
\hline LCIS $(N=1)$ & 0 & 0 & & 0 & & 0 & $1(100 \%)$ \\
\hline Papillary carcinoma $(N=10)$ & $1(10 \%)$ & $2(20 \%)$ & $1(10 \%)$ & $0(0 \%)$ & $1(10 \%)^{\mathrm{a}}$ & $1(10 \%)^{a}$ & $7(70 \%)$ \\
\hline Total $(N=89)$ & $27(30 \%)$ & $27(30 \%)$ & $4(4 \%)$ & $21(24 \%)$ & $2(3 \%)$ & $2(2 \%)$ & $34(38 \%)$ \\
\hline
\end{tabular}

ADH: atypical ductal hyperplasia; CIS: carcinoma in situ; DCIS: ductal carcinoma in situ; LCIS lobular carcinoma in situ.

${ }^{\mathrm{a}}$ Includes one case with NRAS Q61H and PIK3CA exon 13 mutations; thus, seven papillary carcinoma cases were wild type.

domain mutations, with two E542K and two E545K (Tables 1 and 2, Figure 1). Other PIK3CA mutations included one case with a mutation in exon 4 (N345K, C2 domain), and one case with multiple exon 13 kinase domain mutations (Tables 1 and 2). Invasive breast carcinomas with multiple PIK3CA mutations have been previously reported, ${ }^{13-15,17-19}$ though this case appears unique in its mutational profile. Overall, a total of 54 of 89 papillary lesions $(61 \%)$ had mutations in the PIK3CA/AKT pathway.

In addition, two cases had mutations in RAS family proteins, with one HRAS G12D mutation and one NRAS Q61H mutation (Tables 1 and 2, Figure 2). The case with NRAS mutation also had PIK3CA exon 13 mutations, as listed above. Forty-five cases were screened for RAS family mutations, using the full OncoCarta panel, which also encompasses hotspot mutations in $A B L 1, A K T 2, B R A F, C D K$, EGFR, ERBB2, FGFR1, FGFR3, FLT3, KIT, JAK2, KRAS, MET, PDGFRA, and RET; no mutations were identified in any of these other genes.

\section{Correlation of Mutation Status with Histopathologic Features}

The majority of AKT1 mutations were identified in papillomas with no more than mild epithelial hyperplasia; there were 15 such cases among the 27 total AKT1 mutations (56\%). Nevertheless, we did identify $A K T 1$ mutations in papillomas with atypical ductal hyperplasia or ductal carcinoma in situ (three cases each). Only a single carcinoma case was found to have an AKT1 mutation (Tables 1 and 2, Figures 1-3).

In marked contrast to $A K T 1, P I K 3 C A$ exon 20 mutations were preferentially found in papillomas with moderate to florid epithelial hyperplasia. Of 21 PIK3CA exon 20 mutations, 12 were seen in papillomas with hyperplasia $(57 \%)$. Only four papillomas with no to mild hyperplasia had PIK3CA exon 20 mutations (20\%). The difference in frequency of AKT1 mutations vs PIK3CA exon 20 mutations between benign papillomas with and without epithelial hyperplasia was statistically significant $(P=0.0064)$. PIK3CA exon 20 mutations were also identified in five cases of papillomas with atypical hyperplasia $(5 / 11,46 \%)$, but none with ductal carcinoma in situ. PIK3CA exon 20 mutations were not identified in the 10 papillary carcinomas tested (Tables 1 and 2, Figures 1-3). Other PIK3CA hotspot mutations (exons 4, 9, 13) were found distributed among papillomas with and without epithelial hyperplasia, and seen in a single case of papillary carcinoma.

$R A S$ family mutations were screened in 45 of the 89 papillary tumors and were found only in carcinoma and papillary neoplasms with epithelial atypia. RAS mutations were not identified in any benign papillary lesions.

Interestingly, among the 10 carcinomas, only 3 PIK3CA/AKT pathway mutations were identified. The carcinoma with an $A K T 1$ mutation (case 86) had a background of papilloma, as shown by focal residual myoepithelial cells on p63 and calponin immunohistochemical staining. The case with PIK3CA exon 9 mutation was an encapsulated papillary carcinoma. The papillary carcinoma with multiple PIK3CA exon 13 mutations and NRAS mutation had tubulopapillary features and an extensive invasive component. Seven papillary carcinomas were wild type for tested mutations (six screened with full OncoCarta mutation panel and one with HPLC). The percentage of papillary carcinomas with PIK3CA/AKT pathway mutations $(3 / 10,30 \%)$ was considerably lower than in benign or atypical papillary neoplasms. The difference in frequency of combined PIK3CA and AKT1 mutations between papillomas without atypia and papillary carcinomas was statistically significant, despite the relatively small numbers of carcinomas analyzed $(P=0.0425)$. 


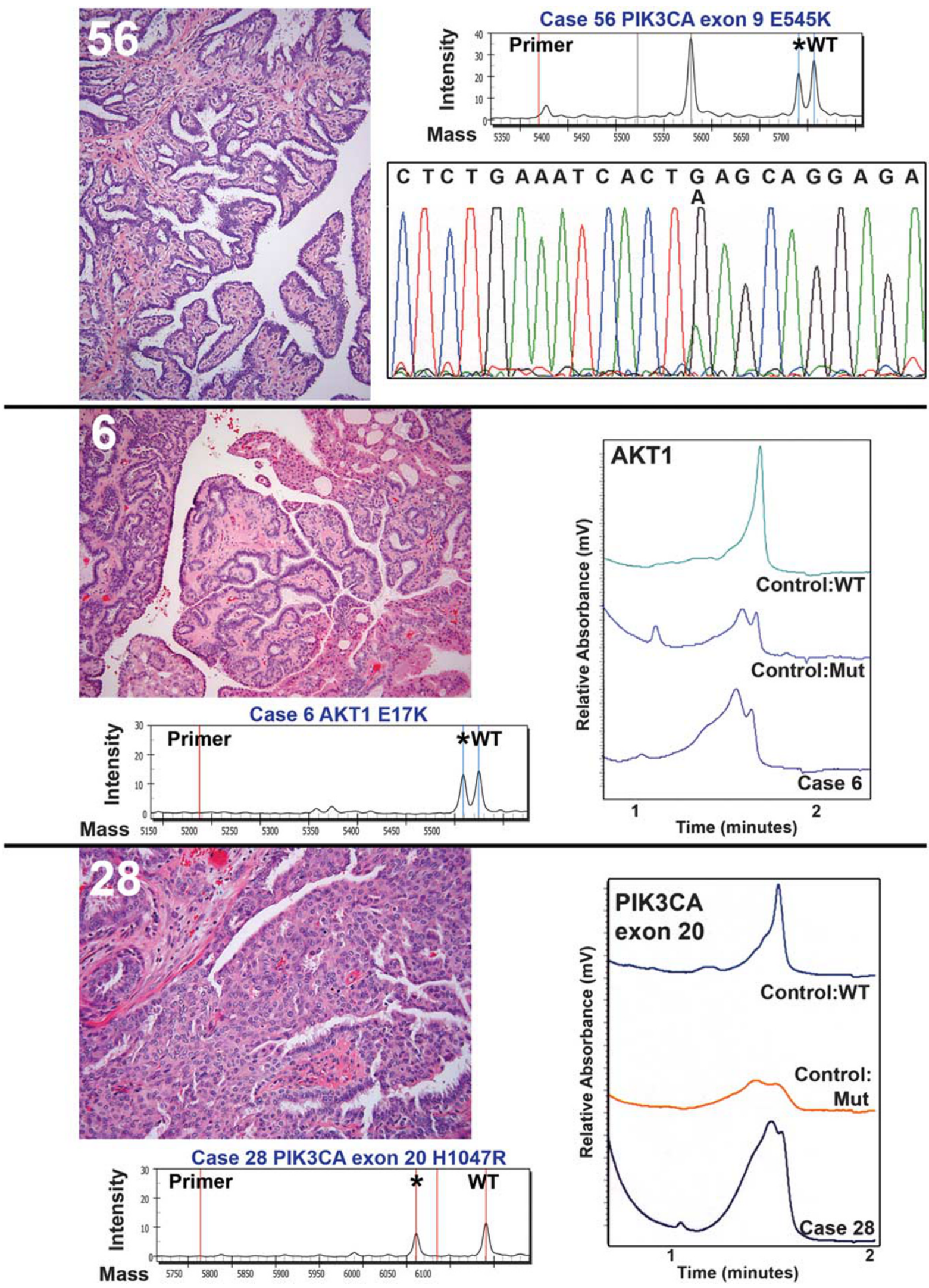


Correlation of Mutational Status and Histopathologic Features with Estrogen Receptor Expression

A subset of papillary neoplasms was stained immunohistochemically for estrogen receptor and scored for percent nuclear positivity $(0-100 \%)$ as well as staining intensity $(0-3+)$. A product score was calculated as the percent positive nuclei multiplied by stain intensity. The average product score did not differ among papillary neoplasms with AKT1 mutation, PIK3CA mutation, or wild-type gene status (Table 3). Further, estrogen receptor staining was also analyzed by histopathologic type of papillary neoplasm and showed the expected variability, with strong uniform staining of papillary neoplasms with atypical ductal hyperplasia and low-grade carcinoma, and variable staining in epithelial hyperplasia (Table 3).

\section{Discussion}

Because precursors of infiltrating breast carcinoma, such as ductal carcinoma in situ, and even atypical ductal hyperplasia, have been shown to exhibit key genetic changes in common with carcinoma, ${ }^{20,33-38}$ we sought to investigate the relationship of papillary lesions to the spectrum of breast premalignant lesions by analyzing kinase mutations. Point mutations in PIK3CA and AKT1 are relatively common in breast carcinoma (average 25 and 4\%, respectively), ${ }^{10-23}$ and are located in a small number of 'hotspots', many of which have transforming capabilities in vitro. ${ }^{21,26,39-41}$ Further, we have previously shown that PIK3CA and AKT1 mutations are present in ductal carcinoma in situ. ${ }^{20}$

In this study, we screened papillary lesions for common mutations in AKT1 and PIK3CA, and found that benign papillomas (defined as papillomas without atypia, ductal carcinoma in situ or carcinoma) have an unexpectedly high frequency of $A K T 1$ mutations $(33 \%, 20 / 61)$. We also found PIK3CA mutations in $33 \%$ of benign papillomas (20/61). Overall, 66\% of benign papillary breast lesions had a PIK3CA or AKT1 mutation (40/61). In contrast, papillary carcinomas have a lower rate of mutation in the PIK3CA/AKT pathway $(30 \% ; P=0.0425)$. The one example of a papillary carcinoma with an AKT1 mutation could have arisen within a background of papilloma, as discerned by the presence of focal residual myoepithelial cells. Because most papillary carcinomas lack PIK3CA or AKT1 mutations, it is unlikely that they could have developed from mutation-positive papillomas. These findings suggest that although some papillary carcinomas may be seen in association with benign papillomas, many other papillary carcinomas may arise through a different molecular pathway. The relationship of papillomas without PIK3CA or AKT1 mutation, if any, to the subsequent evolution of papillary carcinoma or invasive carcinoma remains to be determined.

Likewise, the $30 \%$ frequency of papillary neoplasms with $A K T 1(27 / 89)$ mutations is far greater than has been reported in the literature for infiltrating breast carcinoma (generally on the order of $4 \%)^{19,21-23}$ We also observed a markedly different distribution of PIK3CA mutations in papillary neoplasms, as compared with invasive carcinoma. Meta-analysis of the literature shows that approximately $39 \%$ of PIK3CA mutations in invasive breast carcinoma are exon 9 hotspots (helical domain, E542/5K), 54\% are exon 20 mutations (kinase domain, H1047R/L) and 6\% are mutations at other sites (http://www.sanger.ac.uk/genetics/CGP/cosmic/). ${ }^{13-20}$ In contrast, of PIK3CA mutations in papillary neoplasms, we found that the vast majority were exon $20 \mathrm{H} 1047 \mathrm{R} / \mathrm{L}(77 \%)$ with only $15 \%$ in exon 9. Further, there was an apparent propensity for PIK3CA H1047R/L exon 20 mutations in papillary lesions with moderate to florid epithelial hyperplasia; more than half of the PIK3CA exon 20 mutations were found in this histologic category (Figure 3). We previously identified a PIK3CA H1047R mutation in one of two tested cases of florid usual ductal hyperplasia, from a patient with invasive ductal carcinoma and ductal carcinoma in situ harboring the same mutation. ${ }^{20}$ The place of usual ductal hyperplasia, if any, along the spectrum of premalignant lesions in breast carcinogenesis is also quite controversial. On the basis of molecular data, primarily loss of heterozygosity and comparative genomic hybridization studies, a number of authors have shown clonal chromosomal aberrations in usual ductal hyperplasia, though they occur at lower frequency, with fewer alterations per lesion, as compared with atypical ductal hyperplasia, ductal carcinoma in situ, and invasive carcinomas; other studies have shown identical chromosomal changes in usual ductal hyperplasia and accompanying invasive carcinoma. ${ }^{37,42-48}$ Investigation of activating point mutations in PIK3CA, AKT and other cell signaling pathways in usual ductal

Figure 1 Morphology and mutational analysis of representative nonatypical papillary neoplasms. Case 56 (top): Papilloma with prominent myoepithelial cells and lack of epithelial hyperplasia, as seen in H\&E stain, left panel. Sequenom mutational analysis is at right with mutant peak $\left({ }^{*}\right)$ at PIK3CA exon 9 E545K and exon 9 wild-type peak (WT); large peak in middle at solid gray line is wild-type peak for an unrelated assay. Confirmatory sequencing is below. Case 6 (middle): Papilloma with hyperplasia and apocrine metaplasia, as shown at left with H\&E stain. Below is Sequenom analysis, showing mutant peak (*) for AKT1 exon 2 E17K; confirmatory HPLC WAVE analysis is at right. Case 28 (bottom): Papilloma with florid hyperplasia. Left panel shows H\&E-stained section. Below is Sequenom mutational analysis with mutant peak $\left({ }^{*}\right)$ at PIK3CA exon 20 H1047R. Confirmatory WAVE analysis is at right. (Original magnifications, cases 56 and $6, \times 100$; case $28, \times 200$.) 

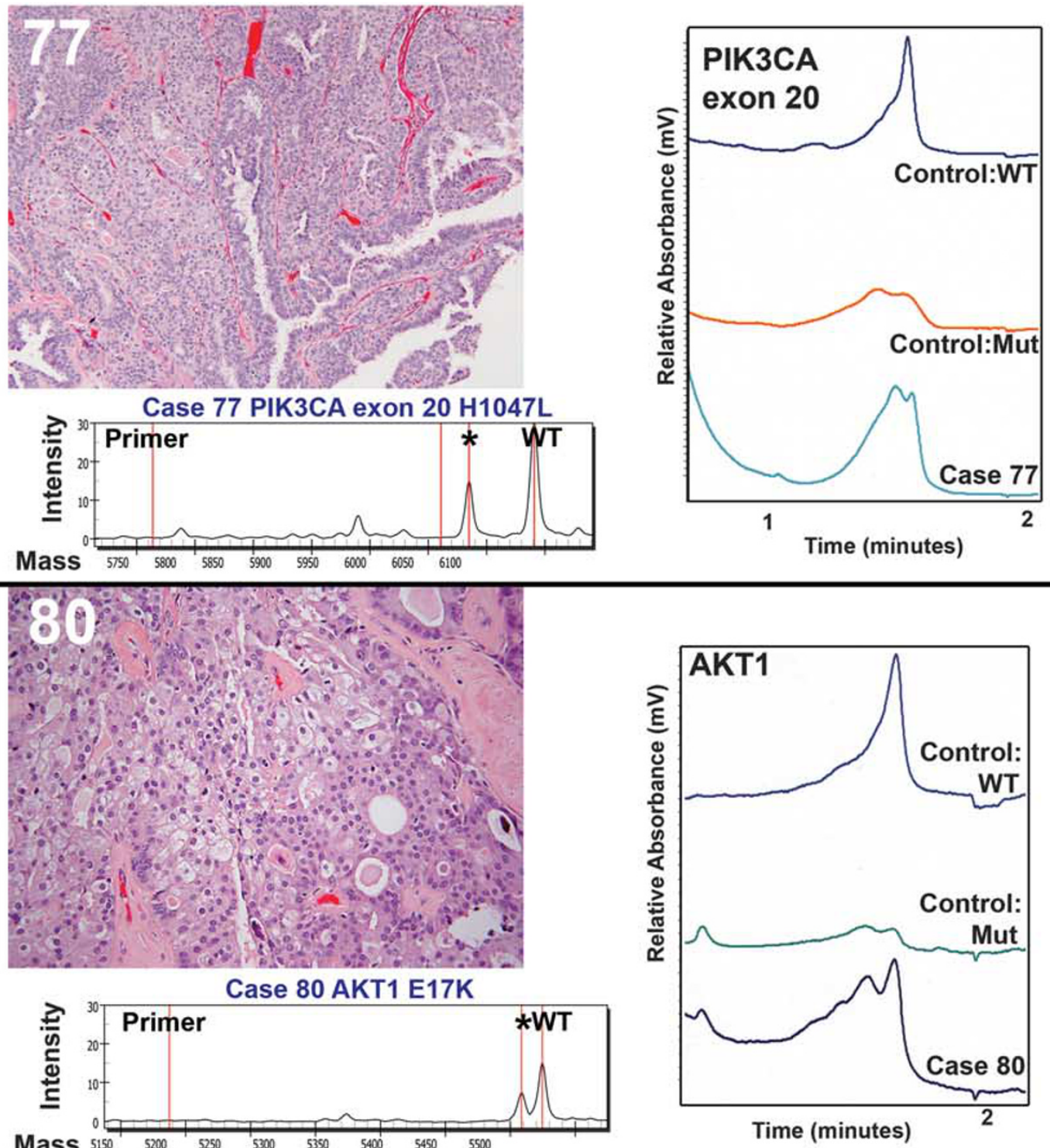

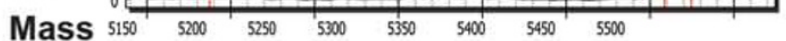

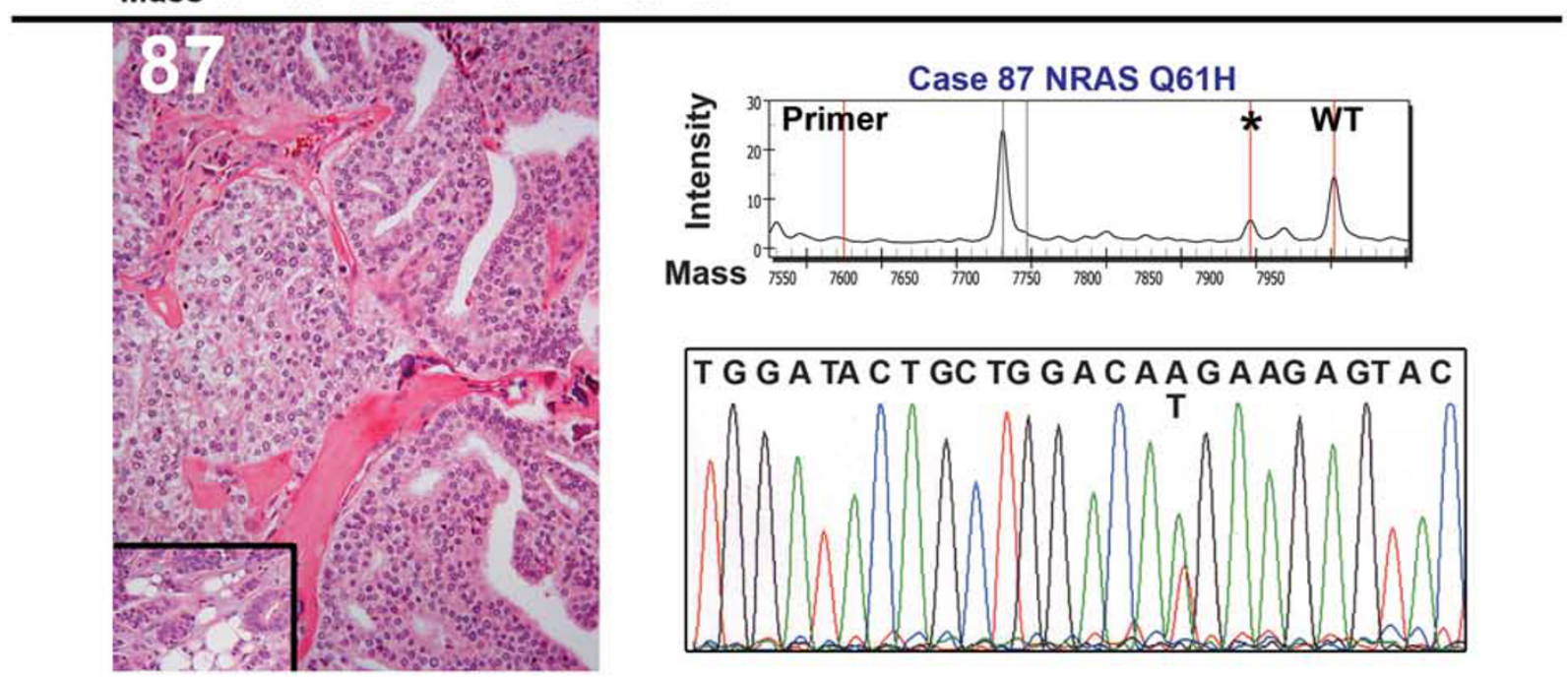


Figure 2 Morphology and mutational analysis of representative papillary neoplasms with atypia or carcinoma. Case 77 (top): Papilloma with atypical ductal hyperplasia. Left panel shows H\&E-stained section; below is Sequenom mutational analysis with mutant peak ( ${ }^{*}$ ) for PIK3CA exon 20 H1047L, and exon 20 wild-type peak (WT), with confirmatory HPLC WAVE analysis at right. Case 80 (middle): Papilloma with ductal carcinoma in situ. Left panel shows H\&E-stained section, below is Sequenom mutational analysis with mutant peak $(*)$ for AKT1 exon 2 E17K, with confirmatory HPLC WAVE analysis at right. Case 87 (bottom): Papillary carcinoma with invasion. Left panel shows H\&E-stained section of papillary carcinoma; inset shows invasive component. At right is Sequenom mutational analysis with mutant peak (*) for NRAS Q61. Large peak at right at solid gray line is wild-type peak for an unrelated assay. Confirmatory direct DNA sequencing is shown below, right. (Original magnifications: case $77, \times 100$; case $80, \times 200$; case $87, \times 200$ with $\times 100$ inset.)

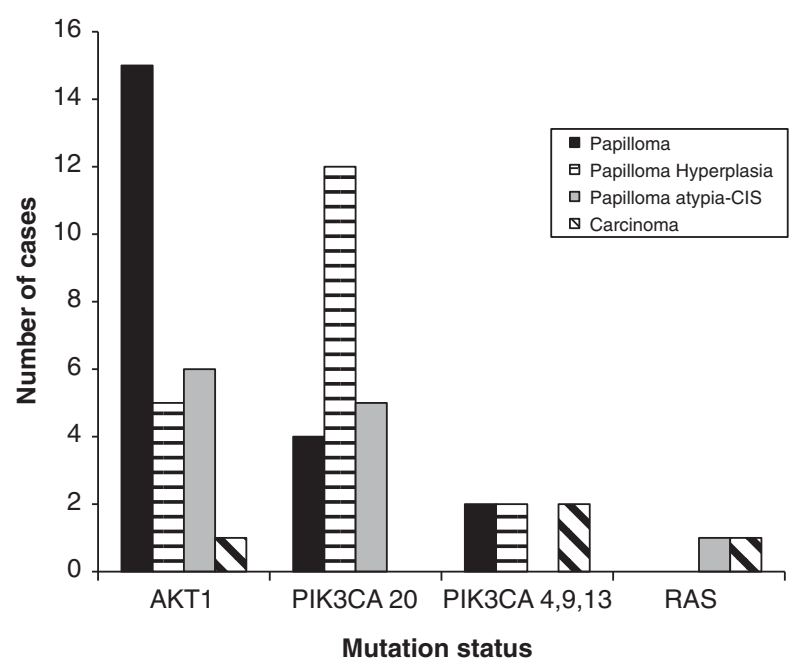

Figure 3 Histopathology of papillary lesions by mutation status. CIS, carcinoma in situ.

Table 3 Estrogen receptor immunohistochemical staining

\begin{tabular}{|c|c|c|c|}
\hline & $\mathrm{N}$ & $\begin{array}{c}\text { Average estrogen } \\
\text { receptor product } \\
\text { score (standard } \\
\text { deviation) }\end{array}$ & $\begin{array}{c}\text { Cases with diffuse } \\
\text { ER staining (\%) }\end{array}$ \\
\hline By mutation status & 34 & & \\
\hline AKT1 mutation & 10 & $185( \pm 105)$ & 50 \\
\hline PIK3CA mutation & 10 & $220( \pm 87)$ & 80 \\
\hline Wild type & 14 & $219( \pm 91)$ & 71 \\
\hline $\begin{array}{l}\text { By histopathologic } \\
\text { type }\end{array}$ & 34 & & \\
\hline Papilloma & 9 & $170( \pm 100)$ & 56 \\
\hline $\begin{array}{l}\text { Papilloma } \\
\text { hyperplasia }\end{array}$ & 10 & $152( \pm 86)$ & $30^{\mathrm{a}}$ \\
\hline $\begin{array}{l}\text { Papilloma } \\
\text { atypia-carcinoma } \\
\text { in situ }\end{array}$ & 8 & $248( \pm 53)$ & $100^{\mathrm{a}}$ \\
\hline $\begin{array}{l}\text { Papillary } \\
\text { carcinoma }\end{array}$ & 7 & $296( \pm 11)$ & $100^{\mathrm{a}}$ \\
\hline
\end{tabular}

Product score $=$ staining intensity $(0-3+)$ multiplied by percent of nuclei staining.

Diffuse estrogen receptor staining defined as $>75 \%$ of nuclei

${ }^{a}$ Statistically significant difference between papilloma with epithelial hyperplasia and papilloma with atypia-carcinoma in situ $(P=0.004)$, and between papilloma with epithelial hyperplasia and papillary carcinoma $(P=0.009)$. Other pair-wise comparisons, including by mutation status, nonsignificant.

hyperplasia, and other breast precursor lesions such as atypical ductal hyperplasia and columnar cell change, should be instructive in further characterizing the divergent molecular and histologic pathways of breast cancer evolution.
Mutations in small GTP binding proteins of the $R A S$ family are relatively rare in breast carcinoma, although $R A S$ signaling is frequently upregulated by other mechanisms. ${ }^{49,50}$ We found two cases with $R A S$ family hotspot mutations, including one papilloma with atypical ductal hyperplasia and one papillary carcinoma. The RAS mutations identified in papillary neoplasms (codon 12, P-loop; codon 61, switch II domain) block GTP turnover and thus lock the protein in the active, GTP-bound state. ${ }^{50}$ Interestingly, in a preliminary report from Esposito et $a l^{51}$, an HRAS Q61R (CAG $\rightarrow$ AAG) mutation was noted in 2 of 3 papillary carcinomas, whereas 10 papillomas (4 with atypia or ductal carcinoma in situ) were negative for mutations in HRAS or NRAS codon 61, as well as codons 12/13 of KRAS, and the BRAF V600E substitution. Taken together, these studies suggest that mutation of $R A S$ family members may play a key role in papillary carcinoma; however, confirmation will require study of larger numbers of cases.

In summary, our data suggest that $A K T 1$ or PIK3CA mutation plays a role in the majority of benign papillary neoplasms of the breast. Further, our findings suggest that papillary carcinomas develop along a molecular pathway somewhat divergent from most benign papillary proliferations.

\section{Acknowledgements}

We acknowledge expert technical assistance of Carolyn Gendron, Emily Justusson, Dylan Nelson, Cara Poage, Linh Matsumura, Jennifer Podolak, and administrative assistance of Sally Ison and Lynne Rowell. This work was supported in part by a VA Merit Review Grant from the Department of Veterans Affairs (MCH) and by a grant from the Oregon Health \& Science University Medical Research Foundation.

\section{Disclosure/conflict of interest}

Christopher Corless has received honoraria and research support from Sequenom, Inc. None of the other authors has conflict of interest to declare.

\section{References}

1 Mulligan AM, O’Malley FP. Papillary lesions of the breast: a review. Adv Anat Pathol 2007;14:108-119. 
2 Noguchi S, Motomura K, Inaji H, et al. Clonal analysis of solitary intraductal papilloma of the breast by means of polymerase chain reaction. Am J Pathol 1994;144: 1320-1325.

3 Noguchi S, Aihara T, Koyama H, et al. Clonal analysis of benign and malignant human breast tumors by means of polymerase chain reaction. Cancer Lett 1995;90:57-63.

4 Tsuda H, Takarabe T, Inazawa J, et al. Detection of numerical alterations of chromosomes 3, 7, 17 and $\mathrm{X}$ in low-grade intracystic papillary tumors of the breast by multi-color fluorescence in situ hybridization. Breast Cancer 1997;4:247-252.

5 Lininger RA, Park WS, Man YG, et al. LOH at 16p13 is a novel chromosomal alteration detected in benign and malignant microdissected papillary neoplasms of the breast. Hum Pathol 1998;29:1113-1118.

6 Di Cristofano C, Mrad K, Zavaglia K, et al. Papillary lesions of the breast: a molecular progression? Breast Cancer Res Treat 2005;90:71-76.

7 Boecker W, Buerger H, Schmitz K, et al. Ductal epithelial proliferations of the breast: a biological continuum? Comparative genomic hybridization and high-molecular-weight cytokeratin expression patterns. J Pathol 2001;195:415-421.

8 Dietrich CU, Pandis N, Teixeira MR, et al. Chromosome abnormalities in benign hyperproliferative disorders of epithelial and stromal breast tissue. Int J Cancer 1995;60:49-53.

9 Komoike Y, Motomura K, Inaji $\mathrm{H}$, et al. Diagnosis of ductal carcinoma in situ (DCIS) and intraductal papilloma using fluorescence in situ hybridization (FISH) analysis. Breast Cancer 2000;7:332-336.

10 Samuels Y, Velculescu VE. Oncogenic mutations of PIK3CA in human cancers. Cell Cycle 2004;3: 1221-1224.

11 Dillon RL, White DE, Muller WJ. The phosphatidyl inositol-3-kinase signaling network: implications for human breast cancer. Oncogene 2007;26:1338-1345.

12 Bachman KE, Argani P, Samuels Y, et al. The PIK3CA gene is mutated with high frequency in human breast cancers. Cancer Biol Ther 2004;3:772-775.

13 Lee JW, Soung YH, Kim SY, et al. PIK3CA gene is frequently mutated in breast carcinomas and hepatocellular carcinomas. Oncogene 2005;24:1477-1480.

14 Li SY, Rong M, Grieu F, et al. PIK3CA mutations in breast cancer are associated with poor outcome. Breast Cancer Res Treat 2006;96:91-95.

15 Liang X, Lau QC, Salto-Tellez M, et al. Mutational hotspot in exon 20 of PIK3CA in breast cancer among Singapore Chinese. Cancer Biol Ther 2006;5: 544-548.

16 Maruyama N, Miyoshi Y, Taguchi T, et al. Clinicopathologic analysis of breast cancers with PIK3CA mutations in Japanese women. Clin Cancer Res 2007;13:408-414.

17 Saal LH, Holm K, Maurer M, et al. PIK3CA mutations correlate with hormone receptors, node metastasis, and ERBB2, and are mutually exclusive with PTEN loss in human breast carcinoma. Cancer Res 2005;65:2554-2559.

18 Perez-Tenorio G, Alkhori L, Olsson B, et al. PIK3CA mutations and PTEN loss correlate with similar prognostic factors and are not mutually exclusive in breast cancer. Clin Cancer Res 2007;13:3577-3584.

19 Stemke-Hale K, Gonzalez-Angulo AM, Lluch A, et al. An integrative genomic and proteomic analysis of
PIK3CA, PTEN, and AKT mutations in breast cancer. Cancer Res 2008;68:6084-6091.

20 Dunlap J, Le C, Shukla A, et al. Phosphatidylinositol-3kinase and AKT1 mutations occur early in breast carcinoma. Breast Cancer Res Treat 2009; doi:10.1007/ s10549-009-0406-1.

21 Carpten JD, Faber AL, Horn C, et al. A transforming mutation in the pleckstrin homology domain of AKT1 in cancer. Nature 2007;448:439-444.

22 Bleeker FE, Felicioni L, Buttitta F, et al. AKT1(E17K) in human solid tumours. Oncogene 2008; doi:10.1038/ onc.2008.170.

23 Kim MS, Jeong EG, Yoo NJ, et al. Mutational analysis of oncogenic AKT E17K mutation in common solid cancers and acute leukaemias. Br J Cancer 2008;98: 1533-1535.

24 Hennessy BT, Smith DL, Ram PT, et al. Exploiting the PI3K/AKT pathway for cancer drug discovery. Nat Rev Drug Discov 2005;4:988-1004.

25 Wymann MP, Marone R. Phosphoinositide 3-kinase in disease: timing, location, and scaffolding. Curr Opin Cell Biol 2005;17:141-149.

26 Zhao L, Vogt PK. Class I PI3K in oncogenic cellular transformation. Oncogene 2008;27:5486-5496.

27 Page DL, Rogers LW. Combined histologic and cytologic criteria for the diagnosis of mammary atypical ductal hyperplasia. Hum Pathol 1992;23:1095-1097.

28 Collins LC, Schnitt SJ. Papillary lesions of the breast: selected diagnostic and management issues. Histopathology 2008;52:20-29.

29 Rosen PP. Papillary Carcinoma. In: Rosen PP (ed). Rosen's Breast Pathology, Vol. 1, 3rd edn. Lippincott Williams \& Wilkins: Philadelphia, PA, 2009, pp 429-448.

30 Maluf HM, Koerner FC. Solid papillary carcinoma of the breast. A form of intraductal carcinoma with endocrine differentiation frequently associated with mucinous carcinoma. Am J Surg Pathol 1995;19:1237-1244.

31 Rosen PP. Ductal hyperplasia: usual and atypical. In: Rosen PP (ed). Rosen's Breast Pathology, Vol. 1, 3rd edn. Lippincott Williams \& Wilkins: Philadelphia, PA, 2009, pp 235-238.

32 Pratilas CA, Hanrahan AJ, Halilovic E, et al. Genetic predictors of MEK dependence in non-small cell lung cancer. Cancer Res 2008;68:9375-9383.

33 Ma XJ, Salunga R, Tuggle JT, et al. Gene expression profiles of human breast cancer progression. Proc Natl Acad Sci USA 2003;100:5974-5979.

34 Reis-Filho JS, Lakhani SR. The diagnosis and management of pre-invasive breast disease: genetic alterations in pre-invasive lesions. Breast Cancer Res 2003;5:313-319.

35 Burstein HJ, Polyak K, Wong JS, et al. Ductal carcinoma in situ of the breast. N Engl J Med 2004;350: 1430-1441.

36 Simpson PT, Reis-Filho JS, Gale T, et al. Molecular evolution of breast cancer. J Pathol 2005;205:248-254.

37 Arpino G, Laucirica R, Elledge RM. Premalignant and in situ breast disease: biology and clinical implications. Ann Intern Med 2005;143:446-457.

38 Moulis S, Sgroi DC. Re-evaluating early breast neoplasia. Breast Cancer Res 2008;10:302.

39 Samuels Y, Wang Z, Bardelli A, et al. High frequency of mutations of the PIK3CA gene in human cancers. Science 2004;304:554

40 Isakoff SJ, Engelman JA, Irie HY, et al. Breast cancerassociated PIK3CA mutations are oncogenic in mammary epithelial cells. Cancer Res 2005;65: 10992-11000. 
41 Ikenoue T, Kanai F, Hikiba Y, et al. Functional analysis of PIK3CA gene mutations in human colorectal cancer. Cancer Res 2005;65:4562-4567.

42 O’Connell P, Pekkel V, Fuqua SA, et al. Analysis of loss of heterozygosity in 399 premalignant breast lesions at 15 genetic loci. J Natl Cancer Inst 1998;90:697-703.

43 Werner M, Mattis A, Aubele M, et al. 20q13.2 amplification in intraductal hyperplasia adjacent to in situ and invasive ductal carcinoma of the breast. Virchows Arch 1999;435:469-472.

44 Aubele MM, Cummings MC, Mattis AE, et al. Accumulation of chromosomal imbalances from intraductal proliferative lesions to adjacent in situ and invasive ductal breast cancer. Diagn Mol Pathol 2000;9:14-19.

45 Allred DC, Mohsin SK, Fuqua SA. Histological and biological evolution of human premalignant breast disease. Endocr Relat Cancer 2001;8:47-61.

46 Gong G, DeVries S, Chew KL, et al. Genetic changes in paired atypical and usual ductal hyperplasia of the breast by comparative genomic hybridization. Clin Cancer Res 2001;7:2410-2414.

47 Jones C, Merrett S, Thomas VA, et al. Comparative genomic hybridization analysis of bilateral hyperplasia of usual type of the breast. J Pathol 2003;199:152-156.

$48 \mathrm{Yu}$ Q, Niu Y, Yu Y, et al. Analysis of the progression of intraductal proliferative lesions in the breast by PCRbased clonal assay. Breast Cancer Res Treat 2009;114: 433-440.

49 Malaney S, Daly RJ. The ras signaling pathway in mammary tumorigenesis and metastasis. J Mammary Gland Biol Neoplasia 2001;6:101-113.

50 Karnoub AE, Weinberg RA. Ras oncogenes: split personalities. Nat Rev Mol Cell Biol 2008;9:517-531.

51 Esposito N, Nikiforova M, Nikiforov Y, et al. Mutational Profile of RAS and BRAF genes in papillary lesions of the breast: identification of a common HRAS mutation in intracystic papillary carcinoma. Mod Pathol 2008;21:47A. 